\title{
CRITICAL NONLINEARITY EXPONENT AND SELF-SIMILAR ASYMPTOTICS FOR LÉVY CONSERVATION LAWS
}

\author{
Piotr BILER $^{\text {a }}$, Grzegorz KARCH ${ }^{\text {a }}$, Wojbor A. WOYCZYŃSKI ${ }^{\mathrm{b}}$ \\ ${ }^{a}$ Instytut Matematyczny, Uniwersytet Wroctawski, pl. Grunwaldzki 2/4, 50-384 Wrocław, Poland \\ ${ }^{\mathrm{b}}$ Department of Statistics and Center for Stochastic and Chaotic Processes in Science and Technology, \\ Case Western Reserve University, Cleveland, OH 44106-7054, USA
}

Received 16 June 2000, revised 2 February 2001

ABSTRACT. - Nonlocal conservation laws of the form

$$
u_{t}+\mathcal{L} u+\nabla \cdot f(u)=0
$$

where $-\mathcal{L}$ is the generator of a Lévy semigroup on $L^{1}\left(\mathbb{R}^{n}\right)$, are encountered in continuum mechanics as model equations with anomalous diffusion. They are generalizations of the classical Burgers equation. We study the critical case when the diffusion and nonlinear terms are balanced, e.g. $\mathcal{L} \sim(-\Delta)^{\alpha / 2}, 1<\alpha<2, f(s) \sim s|s|^{r-1}, r=1+(\alpha-1) / n$. The results include decay rates of solutions and their genuinely nonlinear asymptotic behavior as time $t$ tends to infinity, determined by self-similar source solutions.

(C) 2001 L'Association Publications de l'Institut Henri Poincaré. Published by Elsevier B.V. All rights reserved

1991 MSC: 35K; 35B40; 35Q

Keywords: Generalized Burgers equation; Lévy diffusion; Source solutions; Self-similar asymptotics of solutions; Conservation laws

\section{Introduction and motivation}

The goal of this paper is to study the critical self-similar asymptotics of the Lévy conservation laws which can be written in the form

$$
u_{t}+\mathcal{L} u+\nabla \cdot f(u)=0
$$

where $x \in \mathbb{R}^{n}, t \geqslant 0, u: \mathbb{R}^{n} \times \mathbb{R}^{+} \rightarrow \mathbb{R}, f: \mathbb{R} \rightarrow \mathbb{R}^{n}$ is a nonlinear term, and $-\mathcal{L}$ is the generator of a symmetric, positivity-preserving, Lévy operator semigroup $\mathrm{e}^{-t \mathcal{L}}, t>0$, on $L^{1}\left(\mathbb{R}^{n}\right)$.

E-mail addresses: biler@math.uni.wroc.pl (P. Biler), karch@ math.uni.wroc.pl (G. Karch), waw@po.cwru.edu (W.A. Woyczyński). 
The initial condition

$$
u_{0}(x)=u(x, 0),
$$

which supplements (1.1), is assumed to be an $L^{1}\left(\mathbb{R}^{n}\right)$ function. If $u_{0}(x) \geqslant 0$ is positive a.e. with $\int_{\mathbb{R}^{n}} u_{0}(x) \mathrm{d} x<\infty$, then (1.1) can model an evolution of densities $u$, i.e. $u(x, t) \geqslant 0, \int_{\mathbb{R}^{n}} u(x, t) \mathrm{d} x=\int_{\mathbb{R}^{n}} u_{0}(x) \mathrm{d} x<\infty$ for all $t>0$.

The asymptotic behavior of solutions of the Cauchy problem (1.1)-(1.2) in the noncritical cases has been studied in [6,5].

The operator $\mathcal{L}$ is a pseudodifferential operator defined by the symbol $a=a(\xi) \geqslant 0$, $\widehat{\mathcal{L} v}(\xi)=a(\xi) \widehat{v}(\xi)$. The function $\mathrm{e}^{-t a(\xi)}$ is positive-definite, so the symbol $a(\xi)$ can be represented, as in [6], by the Lévy-Khintchine formula in the Fourier variables (cf. [2, Chapter I, Theorem 1], or [18, Theorem B.2])

$$
a(\xi)=\mathrm{i} b \xi+q(\xi)+\int_{\mathbb{R}^{n}}\left(1-\mathrm{e}^{-\mathrm{i} \eta \xi}-\mathrm{i} \eta \xi \mathbb{1}_{\{|\eta|<1\}}(\eta)\right) \Pi(\mathrm{d} \eta) .
$$

The fundamental nature of the operator $\mathcal{L}$ is clear from the perspective of probability theory. It represents the most general form of generator of a stochastically continuous Markov process with independent and stationary increments. This fact was our basic motivation for the development of the theory presented below and in other related papers.

We assume (with no loss of generality) that $b=0$, i.e., there is no drift; indeed, a shift of the $x$ variable removes the drift term $b$. The function $q(\xi)=\sum_{j, k=1}^{n} q_{j k} \xi_{j} \xi_{k}$ in (1.3) is a quadratic form on $\mathbb{R}^{n}$, and we suppose that $q(\xi) \geqslant 0$ for all $\xi_{j} \in \mathbb{R}^{n}$, i.e. $q$ is positivedefinite in the wide sense. In [6] we considered $q(\xi)=|\xi|^{2}$, which corresponds to the usual Laplacian $-\Delta$ on $\mathbb{R}^{n}$ as the Gaussian part of $\mathcal{L}$. Finally, $\Pi$ is a Borel measure such that $\Pi(\{0\})=0$ and $\int_{\mathbb{R}^{n}} \min \left(1,|\eta|^{2}\right) \Pi(\mathrm{d} \eta)<\infty$.

Eq. (1.1) generalizes the Burgers equation

$$
u_{t}-u_{x x}+\left(u^{2}\right)_{x}=0,
$$

with $x \in \mathbb{R}$ and $t>0$, in three different directions. First, the case of arbitrary dimension $n$ is considered. Second, $-\partial^{2} / \partial x^{2}$ is replaced by a quite general Lévy operator $\mathcal{L}$. Third, instead of the quadratic nonlinearity, arbitrary (sufficiently smooth) function $f$ is considered.

As is well known, the solutions of the Burgers equation (1.4) with the initial condition (1.2) in $L^{1}(\mathbb{R})$ become asymptotically self-similar as $t \rightarrow \infty$, in the following sense: for each $1 \leqslant p \leqslant \infty$

$$
t^{(1-1 / p) / 2}\|u(t)-U(t)\|_{p} \rightarrow 0, \quad \text { as } t \rightarrow \infty,
$$

where the function $U=U_{M}(x, t)$ has an explicit form

$$
U(x, t)=\frac{1}{\sqrt{t}} \exp \left(-x^{2} /(4 t)\right)\left(K-\int_{0}^{x / \sqrt{t}} \exp \left(-z^{2} / 4\right) \mathrm{d} z\right)^{-1}
$$


This function is the, so-called, source solution of (1.4) such that $\int_{\mathbb{R}} U(x, 1) \mathrm{d} x=$ $\int_{\mathbb{R}} u_{0}(x) \mathrm{d} x \equiv M$ with $K=K(M)$, see, e.g., [16]. The initial data for the source solution are attained in the sense of (narrow) convergence of measures; $\lim _{t \rightarrow 0} U(t)=$ $M \delta_{0}$ means that $\lim _{t \rightarrow 0} \int_{\mathbb{R}} U(x, t) \varphi(x) \mathrm{d} x=M \varphi(0)$ for each bounded $\varphi \in C(\mathbb{R})$. This particular solution of (1.4) is self-similar, i.e.

$$
U(x, t)=\frac{1}{\sqrt{t}} U\left(\frac{x}{\sqrt{t}}, 1\right) .
$$

In other words, $U$ is invariant under the parabolic space-time scaling of functions $u \mapsto u_{\lambda}$ defined, for $\lambda>0$, by

$$
u_{\lambda}=\lambda^{u}\left(\lambda x, \lambda^{2} t\right),
$$

that is, $U \equiv U_{\lambda}$ for each $\lambda>0$. Note that this scaling preserves the integrals: $\int_{\mathbb{R}} u_{\lambda} \mathrm{d} x=$ $\int_{\mathbb{R}} u \mathrm{~d} x=M$. Moreover, the convergence property (1.5) can be restated as

$$
\left\|u_{\lambda}(t)-U(t)\right\|_{p} \rightarrow 0 \quad \text { as } \lambda \rightarrow \infty
$$

for each fixed $t>0$.

All these properties can be established using the Hopf-Cole substitution $u=$ $-(\log v)_{x}$ which reduces (1.4) to the linear heat equation. However, recent publications, cf. e.g., [10], [13], [14], [15], [16], [17], [28], developed versatile functional analytic tools to study the long time behavior of solutions of general multidimensional diffusionconvection equations

$$
u_{t}-\Delta u+b \cdot \nabla\left(|u|^{r-1} u\right)=0
$$

in $\mathbb{R}^{n}$ with a real number $r>1, b \in \mathbb{R}^{n}$. For these equations, in general, no explicit analytic solution is known. Rougly speaking, results in those papers describe the asymptotic behavior in the following three cases:

- $r>1+1 / n$, when the asymptotics is linear, i.e.

$$
t^{n(1-1 / p) / 2}\left\|u(t)-M\left(\mathrm{e}^{t \Delta}\right)\right\|_{p} \rightarrow 0 \quad \text { as } t \rightarrow \infty,
$$

where $M=\int_{\mathbb{R}^{n}} u_{0}(x) \mathrm{d} x,\left(\mathrm{e}^{t \Delta}\right)=(4 \pi t)^{-n / 2} \exp \left(-|x|^{2} /(4 t)\right)$ is the fundamental solution of the heat equation;

- $r=1+1 / n$, when

$$
t^{n(1-1 / p) / 2}\|u(t)-U(t)\|_{p} \rightarrow 0 \quad \text { as } t \rightarrow \infty,
$$

where $U(x, t)=t^{-n / 2} U\left(x t^{-1 / 2}, 1\right)$ is the self-similar solution of (1.6) with $U(x, 0)=M \delta_{0}$;

- $1<r<1+1 / n$, when

$$
t^{(n+1)(1-1 / p) /(2 r)}\|u(t)-U(t)\|_{p} \rightarrow 0 \quad \text { as } t \rightarrow \infty,
$$

holds, where (for $b=(0, \ldots, 0,1)) U$ is a particular self-similar solution of the partly viscous conservation law $U_{t}-\Delta_{y} U+\frac{\partial}{\partial x_{n}}\left(|U|^{r-1} U\right)=0$ such that 
$U(x, 0)=M \delta_{0}$ in the sense of measures. Here $x=\left(y, x_{n}\right), y=\left(x_{1}, \ldots, x_{n-1}\right)$, and $\Delta_{y}=\sum_{j=1}^{n-1} \frac{\partial^{2}}{\partial x_{j}^{2}}$.

The first case can be classified as weakly nonlinear, since in this situation the linear diffusion prevails and the nonlinearity is asymptotically negligible. The second, critical case is genuinely nonlinear, when diffusion and the convection are balanced, and the asymptotics is determined by a special solution of a nonlinear equation. Finally, the third case is hyperbolic, since the asymptotics of solutions is determined by solutions of an equation with strong convection and partial dissipation.

The methods introduced in [16] and developed in [10], [13], [14], [15] and [17] are based on scalings of solutions and use in an essential manner tools associated with the diffusive behavior such as the maximum principle and, for the hyperbolic case (1.9), entropy inequalities. However, it should be mentioned that the scaling methods work efficiently also for certain equations for which the maximum principle fails. An example here is the Korteweg-de Vries-Burgers equation which features dispersion and dissipation, see [19]. However, the extension of the usability of these tools in the context of nonlinear and pseudodifferential equations is far from routine. This is the main novelty of the present paper.

Eqs. (1.1) also generalize the fractal Burgers equation

$$
u_{t}+(-\Delta)^{\alpha / 2} u+b \cdot \nabla\left(u|u|^{r-1}\right)=0
$$

with $r>1, b \in \mathbb{R}^{n}$, studied in [3], as well as the one-dimensional multifractal conservation laws

$$
u_{t}-u_{x x}+\sum_{j=1}^{k} a_{j}\left(-\partial^{2} / \partial x^{2}\right)^{\alpha_{j} / 2} u+f(u)_{x}=0,
$$

with $0<\alpha_{j}<2, a_{j}>0$, and a polynomial nonlinearity $f$, considered in [5]. Here, the fractional power of order $\alpha / 2,0<\alpha<2$, of the Laplacian in $\mathbb{R}^{n}$ (or the second derivative $-\partial^{2} / \partial x^{2}$ in $\left.\mathbb{R}\right)$ is the pseudodifferential operator with the symbol $|\xi|^{\alpha}$.

The study of (1.1), and related model equations with nonlocal nonlinearities considered in [8], is motivated by the anomalous diffusion encountered in many physical phenomena. For instance, there are hydrodynamic models with modified dissipativity (obtained as a closure of a system of moment equations, cf. [1]), models of growth of molecular interfaces [22], interacting diffusive particles [8], etc. Two recent volumes in Springer's Lecture Notes in Physics series [24,23] present applications of equations with fractional derivatives and related stochastic differential equations driven by $\alpha$-stable processes to statistical physics, chaos in Hamiltonian mechanics, hydrodynamics, molecular biology and finance mathematics. We studied some probabilistic questions related to such equations in [4]. Various aspects of turbulence models based on the Burgers equation have been discussed in [27].

In [3]-[8] we studied standard mathematical questions concerning (1.1), (1.10), (1.11), including the solvability of the Cauchy problem in various function spaces, uniqueness and regularity of solutions, as well as the large time asymptotics of solutions. Most of the results, except for, e.g., [3, Proposition 6.1] and some sections in [8,4], 
describe asymptotically linear behavior of solutions to those equations, i.e. the situation when the first term of large time asymptotics is a solution of the linearized equation $v_{t}+\mathcal{L} v=0$ for (1.1) with the same initial condition (1.2) $v(x, 0)=u_{0}(x)$. In those cases, only the second term of asymptotics reflects nonlinear effects. Moreover, it was proved for (1.11), and then for (1.1), that the anomalous diffusion dominates the usual Brownian diffusion described by the Laplacian for $t \rightarrow \infty$, cf. [5, Theorem 1.2] and [6, Theorem 5.1].

The present paper studies Eqs. (1.1) in the balanced case of $1<\alpha<2$ and

$$
r=1+(\alpha-1) / n
$$

This is a generalization of the second case (1.8) for (1.6), when the linear and nonlinear terms are of the same importance over the entire time scale $t>0$. Indeed, if the relation (1.12) holds, then Eq. (1.10) written for the rescaled solution

$$
u_{\lambda}(x, t)=\lambda^{n} u\left(\lambda x, \lambda^{\alpha} t\right)
$$

is again the same fractal Burgers equation (1.10).

In this context, one can think about Eq. (1.1) with critical nonlinearity as a true fractal analog of the classical Burgers equation (1.4).

Note that if $\limsup _{s \rightarrow 0}|f(s)| /|s|^{r}<\infty$, for some $r>1+(\alpha-1) / n$, then $\lim _{t \rightarrow \infty} t^{n(1-1 / p) / \alpha}\left\|u(t)-\mathrm{e}^{-t \mathcal{L}} u_{0}\right\|_{p}=0$, i.e., solutions of (1.1) behave as in the weakly nonlinear case, see [6, Theorem 4.1, Remark 5.2].

We will prove that for each mass $M \in \mathbb{R}, 1<\alpha<2$, the equation (1.10) in the balanced case (1.12) has a unique self-similar solution $U=U_{M}, U(x, t)=$ $t^{-n / \alpha} U\left(x t^{-1 / \alpha}, 1\right)$, and $\int_{\mathbb{R}^{n}} U(x, t) \mathrm{d} x=M$ for all $t>0, \int U(x, t) \varphi(x) \mathrm{d} x \rightarrow M \varphi(0)$ as $t \rightarrow 0$ for all bounded $\varphi \in C^{\infty}\left(\mathbb{R}^{n}\right)$ (Theorem 2.1). Such a solution determines the long time behavior of solutions (with the same mass $M$ ) of Eq. (1.1) which "asymptotically" resemble (1.10) (Theorem 2.2). This, loosely speaking, means that we assume that the symbol $a(\xi)$ of the generator $-\mathcal{L}$ of the Lévy semigroup $\mathrm{e}^{-t \mathcal{L}}$ satisfies $a(\xi) \sim|\xi|^{\alpha}$ for $\xi \rightarrow 0$, and the nonlinearity $f$ is such that $f(s) \sim s^{r}$ with $r=1+(\alpha-1) / n$ as $s \rightarrow 0^{+}$.

Assumptions and statement of results can be found in Section 2. Section 3 contains technical lemmas which will be useful in the proofs of theorems mentioned above. The proofs themselves can be found in Sections 4 and 5. The main results of this paper were announced in a brief note [7].

Finally, let us note that our functional framework is that of the Lebesgue $L^{p}\left(\mathbb{R}^{n}\right)$ spaces. We use notation $\|u\|_{p}$ for the $L^{p}\left(\mathbb{R}^{n}\right)$-norms of functions and $W^{k, p}\left(\mathbb{R}^{n}\right)$ for the Sobolev spaces. The operator norm of an operator $A$ from $L^{q}\left(\mathbb{R}^{n}\right)$ to $L^{p}\left(\mathbb{R}^{n}\right)$ is denoted by $\|A\|_{q, p}$. The constants independent of solutions considered and of $t$ (these constants may depend on the initial values) will be denoted by the same letter $C$, even if they may vary from line to line. A standard reference book for facts from the theory of parabolictype equations and interpolation inequalities is [21]. More general function spaces of Besov and Morrey type, interpolation spaces associated with the operator $\mathcal{L}$, etc., are also suitable for studies of (the optimal conditions of) the solvability and asymptotics of solutions of (1.1), cf. [3], [8]. 
Probabilistic aspects of Lévy operators $\mathcal{L}$ and semigroups generated by them are discussed in [2], [18], [20] and [26].

\section{Main results}

Our first goal is to prove the existence and uniqueness of the source solution to the fractal Burgers equation in the critical case (1.12): $r=1+(\alpha-1) / n$ and $1<\alpha<2$, i.e., the existence and uniqueness of a function $u$ satisfying

$$
\begin{aligned}
& u_{t}+(-\Delta)^{\alpha / 2} u+b \cdot \nabla\left(u|u|^{(\alpha-1) / n}\right)=0, \\
& u(x, 0)=M \delta_{0}(x),
\end{aligned}
$$

for $M \in \mathbb{R}$. The initial condition (2.2) is attained in the sense of (narrow) convergence of measures, i.e.,

$$
\lim _{t \rightarrow 0} \int_{\mathbb{R}^{n}} u(x, t) \varphi(x) \mathrm{d} x=M \varphi(0)
$$

for each bounded continuous function $\varphi \in C\left(\mathbb{R}^{n}\right)$; in fact, it suffices to take bounded $C^{\infty}$ functions.

Note that we consider solutions which are not necessarily positive. The proof of Theorem 2.1 is, however, much simpler for solutions of constant sign. The important assumption is that the nonlinearity $f(s)=s|s|^{(\alpha-1) / n}$ in the convection term is an odd function. Therefore, it suffices to consider the case $M \geqslant 0$; indeed, $-u$ solves (2.1) with $-M \delta_{0}$ as the initial condition.

In the sequel, we will also encounter the integral formulation of (1.1)-(1.2), and other nonlinear pseudodifferential equations like (2.1) and (2.4), below, via the Duhamel formula

$$
u(t)=\mathrm{e}^{-t \mathcal{L}} u_{0}-\int_{0}^{t} \nabla \mathrm{e}^{-(t-\tau) \mathcal{L}} \cdot f(u(\tau)) \mathrm{d} \tau .
$$

Solutions of the integral equation (2.3) are called mild solutions of (1.1). They turn out to be weak solutions enjoying some regularity properties, cf. Lemma 3.5, 3.6 and [6, Section 3].

The existence of solutions of the Cauchy problem (1.1)-(1.2) and their properties follow from [6, Theorem 3.1, Proposition 3.1, Corollary 3.1, Remark 3.1]. We note that the Brownian part $-\Delta$ of $\mathcal{L}$ is essential to guarantee regularity of the solutions of this problem, while the jump component of $\mathcal{L}$, which in this paper is meant as the integral term in (1.3), determines the large time behavior of solutions.

Recall that the Cauchy problem for the critical fractal Burgers equation (2.1) has been studied in [3], where a result on the existence of the local in time solutions with suitably small initial data in the space of measures $\mathcal{M}\left(\mathbb{R}^{n}\right)$ has been proved in the critical case (1.12). The size assumption in [3, Proposition 6.1] was formulated in terms of the quantity $\lim \sup _{t \rightarrow 0} t^{(1-1 / \alpha) / r}\left\|\mathrm{e}^{-t(-\Delta)^{\alpha / 2}} u_{0}\right\|_{r}$, which can be viewed as a norm in an interpolation space associated with the operator $(-\Delta)^{\alpha / 2}$. Since the purpose of [3] was 
to give a direct proof of local in time existence of solutions of (1.10) with $u_{0}$ of weak local regularity, we did not attempt to get optimal global existence results for large initial data. The following result gives a complete solution to this problem. The proof can be found in Section 4.

THEOREM 2.1. - The Cauchy problem (2.1)-(2.2) for the n-dimensional fractal Burgers equation with $1<\alpha<2$, critical nonlinearity, and the initial data of the form $M \delta_{0}, M>0$, has a unique solution $U$ which is positive and has the self-similar form

$$
U(x, t)=t^{-n / \alpha} U\left(x t^{-1 / \alpha}, 1\right) .
$$

It turns out that the unique self-similar solution in Theorem 2.1 determines the long time behavior of solutions to a large class of Cauchy problems

$$
\begin{aligned}
& u_{t}+\mathcal{L} u+b \cdot \nabla\left(u|u|^{(\alpha-1) / n}\right)=0, \\
& u(x, 0)=u_{0}(x),
\end{aligned}
$$

for which the Lévy operator $\mathcal{L}$ satisfies the following condition:

- The symbol $a$ of $\mathcal{L}$ has the form

$$
a(\xi)=\ell|\xi|^{\alpha}+k(\xi),
$$

where $\ell>0,1<\alpha<2$, and $k$ is a symbol of another Lévy operator $\mathcal{K}$ such that

$$
\lim _{\xi \rightarrow 0} \frac{k(\xi)}{|\xi|^{\alpha}}=0 .
$$

Without loss of generality (changing the spatial variable $x$ ) we can assume that $\ell=1$.

Remark 2.1. - It is well known that $a(\xi)$, as a symbol of an operator generating a Lévy semigroup, satisfies the bound $0 \leqslant a(\xi) \leqslant C_{a}\left(1+|\xi|^{2}\right)$, for all $\xi \in \mathbb{R}^{n}$ and a constant $C_{a}$. This fact, combined with the assumptions (2.6) and (2.7), gives the inequality

$$
0 \leqslant a(\xi) \leqslant C\left(|\xi|^{\alpha}+|\xi|^{2}\right),
$$

for all $\xi \in \mathbb{R}^{n}$, and another constant $C$. Similarly,

$$
0 \leqslant k(\xi) \leqslant \varepsilon|\xi|^{\alpha}+C(\varepsilon)|\xi|^{2}
$$

holds for each $\varepsilon>0$ and a constant $C(\varepsilon)$.

Example 2.1. - The assumptions (2.6) and (2.7) are fulfilled by multifractal diffusion operators

$$
\mathcal{L}=-a_{0} \Delta+\sum_{j=1}^{k} a_{j}(-\Delta)^{\alpha_{j} / 2}
$$

with $a_{0} \geqslant 0, a_{j}>0,1<\alpha_{j}<2$, and $\alpha=\min _{1 \leqslant j \leqslant k} \alpha_{j}$. 
Our second main result provides asymptotics of general solutions of the Lévy conservation law with balanced nonlinearity. Its proof can be found in Section 5 .

THEOREM 2.2. - Let $u$ be a solution of the Cauchy problem (2.4)-(2.5) for the $n$-dimensional Lévy conservation law with the Lévy diffusion operator $\mathcal{L}$ satisfying assumptions (2.6)-(2.7), and the initial data $u_{0} \in L^{1}\left(\mathbb{R}^{n}\right)$ such that $\int_{\mathbb{R}^{n}} u_{0}(x) \mathrm{d} x=M$. Then, for each $p \in[1, \infty]$,

$$
t^{n(1-1 / p) / \alpha}\|u(t)-U(t)\|_{p} \rightarrow 0 \quad \text { as } t \rightarrow \infty,
$$

where $U=U_{M}$ is the unique self-similar solution of the problem (2.1)-(2.2) with the initial data $M \delta_{0}$ constructed in Theorem 2.1.

Remark 2.2. - For $\alpha=1$, Eq. (2.4) is linear $u_{t}+\mathcal{L} u+b \nabla u=0$. Moreover, the change of variables $x \mapsto x-t b$ allows us to remove the drift term $b \nabla u$. On the other hand, combining Lemmata 3.2 and 3.3 below we obtain that the large time behavior of solutions of the problem $u_{t}+\mathcal{L} u=0, u(x, 0)=u_{0}(x)$, is described by $M\left(\mathrm{e}^{-t(-\Delta)^{1 / 2}}\right)$.

Remark 2.3. - We do not know what are counterparts of Theorems 2.1 and 2.2 for $0<\alpha<1$ because the estimates of $\nabla u$ from Lemma 3.6 below fail for $\alpha<1$. A similar difficulty was already encountered in [3] where, $\alpha \leqslant 1$, questions of uniqueness and regularity of weak solutions to the fractal Burgers equation $u_{t}+\left(-\partial^{2} / \partial x^{2}\right)^{\alpha / 2} u+$ $u u_{x}=0$ were left unresolved.

Remark 2.4. - For $1<r<1+(\alpha-1) / n$, we expect a hyperbolic large time behavior, but a proof of this conjecture will require new methods, completely different from those in $[10,14-17]$.

\section{Technical lemmas}

In this section we gather several technical tools which will be used in the proofs of Theorems 2.1 and 2.2. Some of these estimates are borrowed from [6], but the core of this section is a collection of estimates for rescaled solutions of certain equations, cf., e.g., $[10,16,14,15,17,13]$.

We begin by recalling that, in view of the assumptions (1.3) and (2.6) imposed on the symbol $a(\xi)$, the semigroup $\mathrm{e}^{-t \mathcal{L}}$ generated by the operator $-\mathcal{L}$ is positivity-preserving and satisfies the decay estimates similar to those in [6, Section 2]:

$$
\begin{gathered}
\left\|\mathrm{e}^{-t \mathcal{L}}\right\|_{1, p} \leqslant C t^{-n(1-1 / p) / \alpha}, \\
\left\|\nabla \mathrm{e}^{-t \mathcal{L}}\right\|_{1, p} \leqslant C t^{-n(1-1 / p) / \alpha-1 / \alpha},
\end{gathered}
$$

for each $p \in[1, \infty]$, all $t>0$, and a constant $C=C_{p}$ (cf. Lemma 3.4, below).

Moreover, the bound $[6,(3.15)]$ guarantees that solutions to the nonlinear problem (2.4)-(2.5) with $u_{0} \in L^{1}\left(\mathbb{R}^{n}\right)$ satisfy the estimate

$$
\|u(t)\|_{p} \leqslant C t^{-n(1-1 / p) / \alpha}\left\|u_{0}\right\|_{1},
$$

for all $t>0$, each $p \in[1, \infty]$, and a constant $C=C_{p}$. 
In what follows it is very important that the nonlinear semigroup associated with the nonlinear problem (2.4)-(2.5) is an order-preserving contraction on $L^{1}\left(\mathbb{R}^{n}\right)$. This property is established as part of the next lemma the proof thereof can be found in $[6$, (3.6), Proposition 3.1, Corollary 3.1].

LEMMA 3.1. - Let u, $v$ be solutions of the Cauchy problem for Eq. (1.1) (or (2.4)) with the initial values $u_{0}, v_{0} \in L^{1}\left(\mathbb{R}^{n}\right)$, respectively.

(i) Then, for every $t \geqslant 0$,

$$
\|u(t)-v(t)\|_{1} \leqslant\left\|u_{0}-v_{0}\right\|_{1} .
$$

(ii) If $u_{0}(x) \leqslant v_{0}(x)$ a.e. in $x$, then

$$
u(x, t) \leqslant v(x, t) \text { a.e. in } x, t .
$$

(iii) If, moreover, $u_{0} \in L^{\infty}\left(\mathbb{R}^{n}\right)$, then

$$
\operatorname{ess} \inf u_{0} \leqslant u(x, t) \leqslant \operatorname{ess} \sup u_{0}, \quad \text { a.e. } x, t .
$$

Next two lemmas show how to approximate $\mathrm{e}^{-t \mathcal{L}} u_{0}$ by a multiple of the kernel $\left(\mathrm{e}^{-t(-\Delta)^{\alpha / 2}}\right)$.

Lemma 3.2. - Assume that the symbol $a(\xi)$ satisfies (2.6) and (2.7). For each $p \in[2, \infty]$, and $u_{0} \in L^{1}\left(\mathbb{R}^{n}\right)$,

$$
\lim _{t \rightarrow \infty} t^{n(1-1 / p) / \alpha}\left\|\mathrm{e}^{-t \mathcal{L}} u_{0}-\mathrm{e}^{-t(-\Delta)^{\alpha / 2}} u_{0}\right\|_{p}=0 .
$$

Proof. - The tool here is the Hausdorff-Young inequality

$$
\|\widehat{v}\|_{p} \leqslant C\|v\|_{q},
$$

valid for every $1 \leqslant q \leqslant 2 \leqslant p \leqslant \infty$ such that $1 / p+1 / q=1$. Recall that

$$
\mathrm{e}^{-t \mathcal{L}} u_{0}(x)=(2 \pi)^{-n} \int_{\mathbb{R}^{n}} \mathrm{e}^{-t a(\xi)+\mathrm{i} x \xi} \widehat{u_{0}}(\xi) \mathrm{d} \xi .
$$

By assumption (2.6), for each $\varepsilon>0$ there exists $\delta>0$ such that, for all $|\xi|<\delta$,

$$
\left.\left.|a(\xi)-| \xi\right|^{\alpha}|\leqslant \varepsilon| \xi\right|^{\alpha} .
$$

Hence, for $p \in[2, \infty]$, by the Hausdorff-Young inequality (3.8), we obtain

$$
\begin{aligned}
\left\|\mathrm{e}^{-t \mathcal{L}} u_{0}-\mathrm{e}^{-t(-\Delta)^{\alpha / 2}} u_{0}\right\|_{p}^{q} & \leqslant C \int_{\mathbb{R}^{n}}\left|\mathrm{e}^{-t a(\xi)}-\mathrm{e}^{-t|\xi|^{\alpha}}\right|^{q}\left|\widehat{u_{0}}(\xi)\right|^{q} \mathrm{~d} \xi \\
& \leqslant C \int_{|\xi|<\delta} \ldots \mathrm{d} \xi+C \int_{|\xi| \geqslant \delta} \ldots \mathrm{d} \xi .
\end{aligned}
$$


For $|\xi|<\delta$, in view of (3.9), we bound the integrand on the right hand side of (3.10) by the following quantity

$$
\begin{aligned}
\left|\mathrm{e}^{-t a(\xi)}-\mathrm{e}^{-t|\xi|^{\alpha}}\right|^{q}\left|\widehat{u_{0}}(\xi)\right|^{q} & \leqslant\left.\left.|t a(\xi)-t| \xi\right|^{\alpha}\right|^{q} \mathrm{e}^{-c t|\xi|^{\alpha}}\left\|u_{0}\right\|_{1}^{q} \\
& \leqslant \varepsilon C t^{q}|\xi|^{\alpha q} \mathrm{e}^{-c t|\xi|^{\alpha}}\left\|u_{0}\right\|_{1}^{q} .
\end{aligned}
$$

Hence, by a change of variables, the first term on the right hand side of (3.10) is bounded from above by

$$
\varepsilon C t^{-n / \alpha}\left\|u_{0}\right\|_{1}^{q} \int_{\mathbb{R}^{n}}|\xi|^{q \alpha} \mathrm{e}^{-c|\xi|^{\alpha}} \mathrm{d} \xi
$$

for all $t>0$, and a constant $C$ independent of $t$ and $\varepsilon$. The second term on the right hand side of (3.10) is estimated directly, using the assumption (2.6), by

$$
\int_{|\xi| \geqslant \delta} \mathrm{e}^{-q t|\xi|^{\alpha}} \mathrm{d} \xi\left\|u_{0}\right\|_{1}^{q} .
$$

It is easy to see that, for every $N>0$, this integral tends to 0 faster than $t^{-N}$.

Since $\varepsilon>0$ was arbitrary, using the above estimates we conclude that (3.7) holds true.

Lemma 3.3. - Assume that $u_{0} \in L^{1}\left(\mathbb{R}^{n}\right)$ and $M=\int_{\mathbb{R}^{n}} u_{0}(x) \mathrm{d} x$. Then, for each $p \in[1, \infty]$,

$$
\lim _{t \rightarrow \infty} t^{n(1-1 / p) / \alpha}\left\|\mathrm{e}^{-t(-\Delta)^{\alpha / 2}} u_{0}-M\left(\mathrm{e}^{-t(-\Delta)^{\alpha / 2}}\right)\right\|_{p}=0 .
$$

Proof. - This result is obtained from the inequality

$$
\left\|h * g(\cdot)-\left(\int_{\mathbb{R}^{n}} h(x) \mathrm{d} x\right) g(\cdot)\right\|_{p} \leqslant C\|\nabla g\|_{p}\|h\|_{L^{1}\left(\mathbb{R}^{n},|x| \mathrm{d} x\right)}
$$

valid for each $p \in[1, \infty]$, all $h \in L^{1}\left(\mathbb{R}^{n},|x| \mathrm{d} x\right), g \in C^{1}\left(\mathbb{R}^{n}\right) \cap W^{1,1}\left(\mathbb{R}^{n}\right)$, and a constant $C=C_{p}$ independent of $g$ and $h$. This inequality is a particular case of a more general result proved in [12].

To prove the Lemma we apply (3.11) with $h(x)=u_{0}(x)$ and $g(x)=\left(\mathrm{e}^{-t(-\Delta)^{\alpha / 2}}\right)$, assuming first that $u_{0} \in L^{1}\left(\mathbb{R}^{n},|x| \mathrm{d} x\right)$. The general case of $u_{0} \in L^{1}\left(\mathbb{R}^{n}\right)$ can be handled by an approximation argument. Details of such a reasoning are in [5, Corollaries 2.1 and 2.2].

The following consequence of Lemma 3.2 and 3.3 will be useful in the proof of Theorem 2.2 for $p=\infty$.

COROllary 3.1. - Assume that the symbol a of $\mathcal{L}$ satisfies (2.6) and (2.7). Let $u_{0}$, $v_{0} \in L^{1}\left(\mathbb{R}^{n}\right)$ and $\int_{\mathbb{R}^{n}} u_{0}(x) \mathrm{d} x=\int_{\mathbb{R}^{n}} v_{0}(x) \mathrm{d} x=M$. Then,

$$
\lim _{t \rightarrow \infty} t^{n / \alpha}\left\|\mathrm{e}^{-t \mathcal{L}} u_{0}-\mathrm{e}^{-t(-\Delta)^{\alpha / 2}} v_{0}\right\|_{\infty}=0
$$


Proof. - By the triangle inequality, we have

$$
\begin{aligned}
\left\|\mathrm{e}^{-t \mathcal{L}} u_{0}-\mathrm{e}^{-t(-\Delta)^{\alpha / 2}} v_{0}\right\|_{\infty} \leqslant & \left\|\mathrm{e}^{-t \mathcal{L}} u_{0}-\mathrm{e}^{-t(-\Delta)^{\alpha / 2}} u_{0}\right\|_{\infty} \\
& +\left\|\mathrm{e}^{-t(-\Delta)^{\alpha / 2}} u_{0}-M\left(\mathrm{e}^{-t(-\Delta)^{\alpha / 2}}\right)\right\|_{\infty} \\
& +\left\|M\left(\mathrm{e}^{-t(-\Delta)^{\alpha / 2}}\right)-\mathrm{e}^{-t(-\Delta)^{\alpha / 2}} v_{0}\right\|_{\infty} .
\end{aligned}
$$

An application of Lemmas 3.2 and 3.3 concludes the proof.

Now, for $\lambda>0$, let us consider the rescaled function

$$
u_{\lambda}(x, t) \equiv \lambda^{n} u\left(\lambda x, \lambda^{\alpha} t\right)
$$

where $u$ is a solution to (2.4)-(2.5). Going back to (2.4), one easily checks that $u_{\lambda}$ is a solution of the problem

$$
\begin{aligned}
& \frac{\partial}{\partial t} u_{\lambda}+\mathcal{L}_{\lambda} u_{\lambda}+b \cdot \nabla\left(u_{\lambda}\left|u_{\lambda}\right|^{(\alpha-1) / n}\right)=0, \\
& u_{\lambda}(x, 0)=\lambda^{n} u_{0}(\lambda x) \equiv u_{0, \lambda}(x) .
\end{aligned}
$$

Here, $\mathcal{L}_{\lambda}$ is the rescaled Lévy operator defined by the symbol $\lambda^{\alpha} a(\xi / \lambda)$. In the next lemma, we gather some estimates of the kernel $\left(\mathrm{e}^{-t \mathcal{L}_{\lambda}}\right)$ of the linear semigroup $\mathrm{e}^{-t \mathcal{L}_{\lambda}}$ generated by $-\mathcal{L}_{\lambda}$.

LEMMA 3.4. - Assume that the symbol a $(\xi)$ of the operator $\mathcal{L}$ satisfies assumption (2.6). Then, for every $p \in[1, \infty]$, there exists a constant $C$ independent of $\lambda$ and $t$ such that

$$
\begin{gathered}
\left\|\mathrm{e}^{-t \mathcal{L}_{\lambda}} u_{0}\right\|_{p} \leqslant C t^{-n(1-1 / p) / \alpha}\left\|u_{0}\right\|_{1}, \\
\left\|\nabla \mathrm{e}^{-t \mathcal{L}_{\lambda}} u_{0}\right\|_{p} \leqslant C t^{-n(1-1 / p) / \alpha-1 / \alpha}\left\|u_{0}\right\|_{1}, \\
\left\|\nabla\left(b \cdot \nabla \mathrm{e}^{-t \mathcal{L}_{\lambda}} u_{0}\right)\right\|_{p} \leqslant C t^{-n(1-1 / p) / \alpha-2 / \alpha}\left\|u_{0}\right\|_{1},
\end{gathered}
$$

for all $t>0$.

Proof. - Note that under assumption (2.6) the symbol of the operator $\mathcal{L}_{\lambda}$ satisfies $\lambda^{\alpha} a(\xi / \lambda)=|\xi|^{\alpha}+\lambda^{\alpha} k(\xi / \lambda)$. Now the reasoning is based on the crucial decomposition of the kernels of semigroups

$$
\left(\mathrm{e}^{-t \mathcal{L}_{\lambda}}\right)=\left(\mathrm{e}^{-t(-\Delta)^{\alpha / 2}}\right) *\left(\mathrm{e}^{-t \mathcal{K}_{\lambda}}\right),
$$

where $\mathcal{K}_{\lambda}$ is the Lévy operator corresponding to the symbol $\lambda^{\alpha} k(\xi / \lambda)$. Since $\left\|\left(\mathrm{e}^{-t \mathcal{K}_{\lambda}}\right)\right\|_{1}=1$, it follows immediately from the Young inequality and from the basic estimates of $\left(\mathrm{e}^{-t(-\Delta)^{\alpha / 2}}\right)$ that

$$
\left\|\left(\mathrm{e}^{-t \mathcal{L}_{\lambda}}\right)\right\|_{p} \leqslant C\left\|\left(\mathrm{e}^{-t(-\Delta)^{\alpha / 2}}\right)\right\|_{p}\left\|\left(\mathrm{e}^{-t \mathcal{K}_{\lambda}}\right)\right\|_{1} \leqslant C t^{-n(1-1 / p) / \alpha} .
$$

The proof of (3.15) is analogous but (3.17) has to be replaced by

$$
\nabla\left(\mathrm{e}^{-t \mathcal{L}_{\lambda}}\right)=\nabla\left(\mathrm{e}^{-t(-\Delta)^{\alpha / 2}}\right) *\left(\mathrm{e}^{-t \mathcal{K}_{\lambda}}\right) .
$$


The bound (3.16) is obtained in the same manner.

In what follows we will need some estimates of $u_{\lambda}$ which are uniform with respect to $\lambda \geqslant 1$.

LEMMA 3.5. - Assume that $u$ is a solution to (2.4)-(2.5) with the initial data $u_{0} \in L^{1}\left(\mathbb{R}^{n}\right)$, and the rescaled function $u_{\lambda}$ is given by (3.12). Then, for every $p \in[1, \infty]$, there exists a constant $C$, independent of $\lambda, t$, such that

$$
\left\|u_{\lambda}(t)\right\|_{p} \leqslant C t^{-n(1-1 / p) / \alpha}\left\|u_{0}\right\|_{1}
$$

for all $\lambda>0, t>0$.

Proof. - This follows from (3.3) by a simple change of variables:

$$
\begin{aligned}
\left\|u_{\lambda}(t)\right\|_{p} & =\lambda^{n(1-1 / p)}\left\|u\left(\lambda^{\alpha} t\right)\right\|_{p} \leqslant C \lambda^{n(1-1 / p)}\left(\lambda^{\alpha} t\right)^{-n(1-1 / p) / \alpha}\left\|u_{0}\right\|_{1} \\
& =C t^{-n(1-1 / p) / \alpha}\left\|u_{0}\right\|_{1} .
\end{aligned}
$$

for all $\lambda>0, t>0$, and a constant $C$ independent of $\lambda, t$ and $u_{0}$.

LEMMA 3.6. - Under the assumptions of Lemma 3.5, for every $p \in[1, \infty]$, there exists a constant $C$ independent of $\lambda, t$, but, in general, dependent on the initial data $u_{0}$, such that

$$
\left\|\nabla u_{\lambda}(t)\right\|_{p} \leqslant C t^{-n(1-1 / p) / \alpha-1 / \alpha} .
$$

Proof. - To obtain this bound for $\nabla u_{\lambda}$, we represent $u_{\lambda}$ in the mild form analogous to that in (2.3), but now for the rescaled Eq. (3.13). Next, applying the gradient $\nabla$ to this integral equation, we obtain

$$
\nabla u_{\lambda}(t+1)=\nabla \mathrm{e}^{-t \mathcal{L}_{\lambda}} u_{\lambda}(1)-\int_{0}^{t} \nabla \mathrm{e}^{-(t-\tau) \mathcal{L}_{\lambda}}\left(b \cdot \nabla\left(u_{\lambda}\left|u_{\lambda}\right|^{(\alpha-1) / n}\right)\right)(\tau+1) \mathrm{d} \tau .
$$

Recall that, by (3.15) and (3.18), there is a constant $C>0$ such that, for all $\lambda>0$ and $t>0$,

$$
\left\|\nabla \mathrm{e}^{-t \mathcal{L}_{\lambda}} u_{\lambda}(1)\right\|_{p} \leqslant C t^{-n(1-1 / p) / \alpha-1 / \alpha}\left\|u_{0}\right\|_{1} .
$$

Next, for $\tau \in[0,1]$, using (3.16) and (3.18), we estimate the $L^{p}$-norm of the integrand in (3.20) by the following quantity

$$
\begin{aligned}
& \left\|\nabla\left(b \cdot \nabla\left(\mathrm{e}^{-(t-\tau) \mathcal{L}_{\lambda}}\right)\right)\right\|_{1}\left\|u_{\lambda}(\tau+1)\right\|_{p((\alpha-1) / n+1)}^{(\alpha-1) / n+1} \\
& \quad \leqslant C(t-\tau)^{-2 / \alpha}(\tau+1)^{-n((\alpha-1) / n+1-1 / p) / \alpha} \leqslant C(t-\tau)^{-2 / \alpha},
\end{aligned}
$$

for all $t>1$, and a constant $C$ independent of $t$ and $\lambda$. If $\tau \in[1, t]$, we proceed as follows

$$
\begin{aligned}
& \left\|\nabla \mathrm{e}^{-(t-\tau) \mathcal{L}_{\lambda}}\left(b \cdot \nabla\left(u_{\lambda}\left|u_{\lambda}\right|^{(\alpha-1) / n}\right)\right)(\tau+1)\right\|_{p} \\
& \quad \leqslant C(t-\tau)^{-1 / \alpha}\left\|u_{\lambda}(\tau+1)\right\|_{\infty}^{(\alpha-1) / n}\left\|\nabla u_{\lambda}(\tau+1)\right\|_{p} \\
& \quad \leqslant C(t-\tau)^{-1 / \alpha}\left\|\nabla u_{\lambda}(\tau+1)\right\|_{p} .
\end{aligned}
$$


Here, we have used the following consequence of (3.18):

$$
\left\|u_{\lambda}(\tau+1)\right\|_{\infty} \leqslant C(\tau+1)^{-n / \alpha} \leqslant C .
$$

Hence, computing the $L^{p}$-norm of the expressions in (3.20), and using the above estimates for the integrand in the second term on the right hand side of (3.20), we obtain

$$
\begin{aligned}
\left\|\nabla u_{\lambda}(t+1)\right\|_{p} \leqslant & C\left(t^{-n(1-1 / p) / \alpha-1 / \alpha}+t^{-2 / \alpha+1}+(t-1)^{-2 / \alpha+1}\right) \\
& +C \int_{1}^{t}(t-\tau)^{-1 / \alpha}\left\|\nabla u_{\lambda}(\tau+1)\right\|_{p} \mathrm{~d} \tau .
\end{aligned}
$$

Since $-2 / \alpha+1>-1$ for $\alpha>1$, we may apply the generalized singular Gronwall lemma (cf., e.g., [9, Lemma 1.1]) for $t>1$ to prove the bound $\left\|\nabla u_{\lambda}(t+1)\right\|_{p} \leqslant C(t)$ with a continuous function $C$ on $[0, \infty)$, uniformly with respect to $\lambda>0$. However, by a change of variables, we have

$$
\left\|\nabla u_{\lambda}(t+1)\right\|_{p}=\lambda^{n(1-1 / p)+1}\left\|\nabla u\left(\lambda^{\alpha}(t+1)\right)\right\|_{p} .
$$

Thus, fixing $t=t_{0}>0$ and choosing $\lambda=\left(\frac{t}{t_{0}+1}\right)^{1 / \alpha}$, we obtain

$$
\|\nabla u(t)\|_{p} \leqslant C t^{-n(1-1 / p) / \alpha-1 / \alpha},
$$

for all $t>0$, and a constant $C$ independent of $t$. Finally, observe that the inequality (3.21) allows us to obtain, proceeding as for (3.18), the bound (3.19) for $\nabla u_{\lambda}$.

Next, we will establish some compactness properties of the family of rescaled solutions $\left\{u_{\lambda}\right\}$.

LEMMA 3.7. - There exist a sequence $\lambda_{k} \rightarrow \infty$ and a function $\bar{u}(x, t)$ such that, for every $\eta>0,1<p<\infty, 0<t_{1}<t_{2}<\infty$, and each bounded domain $G \subset \mathbb{R}^{n}$,

$$
\begin{aligned}
& u_{\lambda_{k}} \rightarrow \bar{u} \quad \text { in } C\left(\left[t_{1}, t_{2}\right] ; W^{-2-\eta, p}(G)\right), \\
& \frac{\partial}{\partial t} u_{\lambda_{k}}(t) \rightarrow \frac{\partial}{\partial t} \bar{u}(t) \quad \text { in } \mathcal{D}^{\prime}\left((0, \infty) \times \mathbb{R}^{n}\right) .
\end{aligned}
$$

Moreover, the convergence

$$
u_{\lambda_{k}}(t) \rightarrow \bar{u}(t) \quad \text { in } L_{l o c}^{p}\left(\mathbb{R}^{n}\right)
$$

holds for every $p \in[1, \infty)$ and each $t>0$, and

$$
u_{\lambda_{k}} \rightarrow \bar{u} \quad \text { pointwise a.e. in } \mathbb{R}^{n} \times(0, \infty) .
$$

Proof. - The estimates (3.18) and (3.19) imply that the families $\left\{u_{\lambda}\right\}_{\lambda>0}$ and $\left\{\nabla u_{\lambda}\right\}_{\lambda>0}$ are uniformly bounded in $L_{l o c}^{\infty}\left((0, \infty) ; L^{p}\left(\mathbb{R}^{n}\right)\right)$ for every $p \in[1, \infty]$. The same conclusion holds true for $\left\{b \cdot \nabla\left(u_{\lambda}\left|u_{\lambda}\right|^{(\alpha-1) / n}\right)\right\}_{\lambda>0}$ in view of the inequality

$$
\left\|b \cdot \nabla\left(u_{\lambda}\left|u_{\lambda}\right|^{(\alpha-1) / n}\right)(t)\right\|_{p} \leqslant C\left\|\nabla u_{\lambda}(t)\right\|_{p}\left\|u_{\lambda}(t)\right\|_{\infty}^{(\alpha-1) / n} .
$$


Moreover, using Eq. (3.13) we deduce that $\left\{\frac{\partial}{\partial t} u_{\lambda}\right\}_{\lambda>0}$ is uniformly bounded in $L_{l o c}^{\infty}\left((0, \infty) ; W^{-2, p}\left(\mathbb{R}^{n}\right)\right)$. Taking into account that $W^{s, p}(G)$ is compactly embedded in $W^{r, p}(G)$ for every bounded domain $G$ of $\mathbb{R}^{n}$ and $r<s$, and applying classical compactness argument for vector-valued functions (cf. [25, Corollary 4, p. 85]), we see that $\left\{u_{\lambda}\right\}_{\lambda \geqslant 1}$ is relatively compact in $C\left(\left[t_{1}, t_{2}\right] ; W^{-2-\eta, p}(G)\right)$ for each $G, \eta>0$ and all $0<t_{1}<t_{2}<\infty$. So, there exists a sequence $\lambda_{k} \rightarrow \infty$ and a function $\bar{u}$ such that (3.22) holds, as well as (3.23). As a consequence of (3.18) and (3.19) we obtain also that $\left\{u_{\lambda}(t)\right\}_{\lambda \geqslant 1}$ is relatively compact in $L_{l o c}^{p}\left(\mathbb{R}^{n}\right)$ for every $p \in[1, \infty)$ and each $t>0$. Then the diagonal method allows us to extract a sequence $\lambda_{k} \rightarrow \infty$ such that (3.24) is valid. Again, extracting a subsequence by the diagonal method leads to a subsequence (still denoted by $\left\{\lambda_{k}\right\}_{k \in \mathbb{N}}$ ) such that the last conclusion (3.25) of Lemma 3.7 is satisfied.

LEMMA 3.8. - The function $\bar{u}$ constructed in Lemma 3.7 satisfies Eq. (2.1) in the sense of distributions. Moreover, $\bar{u} \in L^{\infty}\left((0, \infty) ; L^{1}\left(\mathbb{R}^{n}\right)\right)$ and $\|\bar{u}(t)\|_{1} \leqslant\left\|u_{0}\right\|_{1}$ for all $t>0$.

Proof. - In order to pass to the weak limit in Eq. (3.13), we multiply it by an arbitrary test function $\varphi \in C_{c}^{\infty}\left(\mathbb{R}^{n}\right)$ and integrate over $\mathbb{R}^{n}$ :

$$
\begin{array}{r}
\int_{\mathbb{R}^{n}} \frac{\partial}{\partial t} u_{\lambda}(x, t) \varphi(x) \mathrm{d} x+\int_{\mathbb{R}^{n}} \mathcal{L}_{\lambda} u_{\lambda}(x, t) \varphi(x) \mathrm{d} x \\
-\int_{\mathbb{R}^{n}}\left(u_{\lambda}\left|u_{\lambda}\right|^{(\alpha-1) / n}\right)(x, t) b \cdot \nabla \varphi(x) \mathrm{d} x=0 .
\end{array}
$$

It follows from (3.23) that along the sequence $\lambda_{k} \rightarrow \infty$

$$
\int_{\mathbb{R}^{n}} \frac{\partial}{\partial t} u_{\lambda}(x, t) \varphi(x) \mathrm{d} x \rightarrow \int_{\mathbb{R}^{n}} \frac{\partial}{\partial t} \bar{u}(x, t) \varphi(x) \mathrm{d} x .
$$

In order to pass to the limit in the second term in (3.26), we use the decomposition (2.6) which implies

$$
\int_{\mathbb{R}^{n}} \mathcal{L}_{\lambda} u_{\lambda}(x, t) \varphi(x) \mathrm{d} x=\int_{\mathbb{R}^{n}}(-\Delta)^{\alpha / 2} u_{\lambda}(x, t) \varphi(x) \mathrm{d} x+\int_{\mathbb{R}^{n}} \mathcal{K}_{\lambda} u_{\lambda}(x, t) \varphi(x) \mathrm{d} x .
$$

In view of (3.22), the first integral term on the right hand side tends to $\int_{\mathbb{R}^{n}}(-\Delta)^{\alpha / 2} \bar{u}(x, t)$ $\times \varphi(x) \mathrm{d} x$ as $\lambda_{k} \rightarrow \infty$. To deal with the second term, we use the Schwarz inequality, Lemma 3.5, and (2.9) to get

$$
\begin{aligned}
\left|\int_{\mathbb{R}^{n}} \mathcal{K}_{\lambda} u_{\lambda}(x, t) \varphi(x) \mathrm{d} x\right| & \leqslant C\left\|u_{\lambda}(t)\right\|_{2}\left(\int_{\mathbb{R}^{n}}\left|\lambda^{\alpha} k(\xi / \lambda) \widehat{\varphi}(\xi)\right|^{2} \mathrm{~d} \xi\right)^{1 / 2} \\
& \leqslant C t^{-n /(2 \alpha)}\left(\varepsilon^{2}\left\|(-\Delta)^{\alpha / 2} \varphi\right\|_{2}^{2}+\lambda^{\alpha-2} C^{2}(\varepsilon)\|\Delta \varphi\|_{2}^{2}\right)^{1 / 2} .
\end{aligned}
$$

Now, since $\varepsilon$ was arbitrary, we conclude that $\int_{\mathbb{R}^{n}} \mathcal{K}_{\lambda} u_{\lambda}(x, t) \varphi(x) \mathrm{d} x \rightarrow 0$ as $\lambda_{k} \rightarrow \infty$. 
We can apply the Dominated Convergence Theorem and (3.25) in the nonlinear term, because, by (3.18),

$$
\left|u_{\lambda}(x, t)\right|^{(\alpha-1) / n+1}|b \cdot \nabla \varphi(x)| \leqslant C t^{-1-(n-1) / \alpha}|b \cdot \nabla \varphi(x)|,
$$

with $C$ independent of $\lambda$. This proves that $\bar{u}$ satisfies the fractal Burgers equation (2.1) in the sense of distributions.

Now let us observe that by (3.18), $\left\|u_{\lambda}(t)\right\|_{1} \leqslant\left\|u_{0}\right\|_{1}$. Consequently, applying the Fatou Lemma to the sequence of functions $\left|u_{\lambda_{k}}(x, t)\right|$ and using (3.25) we deduce $\|\bar{u}(t)\|_{1} \leqslant\left\|u_{0}\right\|_{1}$ for all $t>0$. Therefore, it follows that $\bar{u} \in L^{\infty}\left((0, \infty) ; L^{1}\left(\mathbb{R}^{n}\right)\right)$.

LEMMA 3.9. - For each function $\varphi \in C_{c}^{\infty}\left(\mathbb{R}^{n}\right)$ with compact support,

$$
\int_{\mathbb{R}^{n}} \bar{u}(x, t) \varphi(x) \mathrm{d} x \rightarrow M \varphi(0) \quad \text { as } t \rightarrow 0 .
$$

Proof. - Observe that, for each smooth function $\varphi \in C_{c}^{\infty}\left(\mathbb{R}^{n}\right)$ with compact support, the estimate

$$
\left\|\mathcal{L}_{\lambda} \varphi\right\|_{\infty} \leqslant C \int_{\mathbb{R}^{n}}\left(|\xi|^{\alpha}+|\xi|^{2}\right)|\widehat{\varphi}(\xi)| \mathrm{d} \xi
$$

holds uniformly in $\lambda \geqslant 1$. Indeed, $\mathcal{L}_{\lambda} \varphi(x)=\int_{\mathbb{R}^{n}} \lambda^{\alpha} a(\xi / \lambda) \widehat{\varphi}(\xi) \mathrm{e}^{\mathrm{i} x \xi} \mathrm{d} \xi$, so that the inequality (3.28) follows from the bound (2.8) on the symbol of $\mathcal{L}$.

We multiply (3.13) by any function $\varphi \in C_{c}^{\infty}\left(\mathbb{R}^{n}\right)$ and integrate over $\mathbb{R}^{n} \times(s, t)$, $0<s<t<\infty$. We are allowed to integrate by parts, which leads to

$$
\begin{aligned}
& \left|\int_{\mathbb{R}^{n}} u_{\lambda}(x, t) \varphi(x) \mathrm{d} x-\int_{\mathbb{R}^{n}} u_{\lambda}(x, s) \varphi(x) \mathrm{d} x\right| \\
& \leqslant \int_{s} \int_{\mathbb{R}^{n}}\left|u_{\lambda}(x, \tau)\right|\left|\mathcal{L}_{\lambda} \varphi(x)\right| \mathrm{d} x \mathrm{~d} \tau \\
& \quad+\int_{s} \int_{\mathbb{R}^{n}}\left|u_{\lambda}(x, \tau)\right|^{(\alpha-1) / n+1}|b \cdot \nabla \varphi(x)| \mathrm{d} x \mathrm{~d} \tau \\
& \leqslant C_{1}|t-s|\left\|\mathcal{L}_{\lambda} \varphi\right\|_{\infty}\left\|u_{0}\right\|_{1}+C_{2}|t-s|^{1 / \alpha}\|\nabla \varphi\|_{\infty},
\end{aligned}
$$

where the constants $C_{1}, C_{2}$ are independent of $\lambda \geqslant 1$ and $0<s<t<\infty$. Here we have used (3.18) with $p=1$, and (3.28), in the first term on the right hand side of (3.29), and (3.18) with $p=(\alpha-1) / n+1$, in the second term.

Passing to the limit $s \rightarrow 0$ in (3.29), we see that estimate (3.29) holds for $s=0$, too. Hence, letting $\lambda_{k} \rightarrow \infty$ in (3.29) with $s=0$, we obtain

$$
\left|\int_{\mathbb{R}^{n}} \bar{u}(x, t) \varphi(x) \mathrm{d} x-M \varphi(0)\right| \leqslant C t+C t^{1 / \alpha} .
$$


Now the passage to the limit $t \rightarrow 0$ gives the desired result (3.27).

LEMMA 3.10. - The relation

$$
\int_{|x| \geqslant R}\left|u_{\lambda}(x, t)\right|^{p} \mathrm{~d} x \rightarrow 0 \quad \text { as } R \rightarrow \infty
$$

holds for every $t>0$, uniformly with respect to $\lambda \geqslant 1$.

Proof. - Let us fix $\psi \in C^{\infty}\left(\mathbb{R}^{n}\right)$ such that $\psi(x)=0$ for $|x| \leqslant 1$, and $\psi(x)=1$ for $|x| \geqslant 2$. For simplicity of notation we shall write $\psi_{R}(x)=\psi(x / R)$. It suffices to prove that

$$
\left\|u_{\lambda}(t) \psi_{R}\right\|_{p} \rightarrow 0 \quad \text { as } R \rightarrow \infty,
$$

uniformly with respect to $\lambda \geqslant 1$ and $0<t_{1} \leqslant t \leqslant t_{2}<\infty$. Without loss of generality, we may assume that $u_{\lambda}$ is nonnegative. Indeed, by the pointwise comparison principle for solutions of (1.1) (cf. Lemma 3.1), we have $\left|u_{\lambda}(x, t)\right| \leqslant v_{\lambda}(x, t)$ for almost all $x \in \mathbb{R}^{n}$ and $t \geqslant 0$, where $v_{\lambda}$ is the solution of (3.13) with the initial data $\left|u_{0, \lambda}\right|$. Hence $\left\|u_{\lambda}(t) \psi_{R}\right\|_{p} \leqslant\left\|v_{\lambda}(t) \psi_{R}\right\|_{p}$.

First consider $p=1$. Multiplying Eq. (3.13) for $u_{\lambda}$ by $\psi_{R}$ and integrating over $\mathbb{R}^{n}$ we have

$$
\begin{aligned}
& \frac{\mathrm{d}}{\mathrm{d} t} \int_{\mathbb{R}^{n}} u_{\lambda}(x, t) \psi_{R}(x) \mathrm{d} x \\
& \quad=-\int_{\mathbb{R}^{n}} \mathcal{L}_{\lambda} u_{\lambda}(x, t) \psi_{R}(x) \mathrm{d} x-\int_{\mathbb{R}^{n}} b \cdot \nabla\left(u_{\lambda}|u|^{(\alpha-1) / n}\right)(x, t) \psi_{R}(x) \mathrm{d} x .
\end{aligned}
$$

Applying the reasoning in the proof of (3.28) to the function $\psi_{R}-1 \in C_{c}^{\infty}\left(\mathbb{R}^{n}\right)$ we obtain that, for $\lambda \geqslant 1$,

$$
\left\|\mathcal{L}_{\lambda} \psi_{R}\right\|_{\infty}=\left\|\mathcal{L}_{\lambda}\left(\psi_{R}-1\right)\right\|_{\infty} \leqslant C \int_{\mathbb{R}^{n}}\left(R^{-\alpha}|\xi|^{\alpha}+R^{-2}|\xi|^{2}\right)\left|\left(\psi_{R}-1\right) \Upsilon(\xi)\right| \mathrm{d} \xi
$$

and, in view of (3.18),

$$
\left|\int_{\mathbb{R}^{n}} \mathcal{L}_{\lambda} u_{\lambda}(x, t) \psi_{R}(x) \mathrm{d} x\right| \leqslant\left\|\mathcal{L}_{\lambda} \psi_{R}\right\|_{\infty} \int_{\mathbb{R}^{n}}\left|u_{\lambda}(x, t)\right| \mathrm{d} x \leqslant C\left(R^{-\alpha}+R^{-2}\right)\left\|u_{0}\right\|_{1} .
$$

The integration by parts and (3.18) give

$$
\begin{aligned}
\left|\int_{\mathbb{R}^{n}} b \cdot \nabla\left(u_{\lambda}|u|^{(\alpha-1) / n}\right)(x, t) \psi_{R}(x) \mathrm{d} x\right| & \leqslant\left\|b \cdot \nabla \psi_{R}\right\|_{\infty} \int_{\mathbb{R}^{n}}\left|u_{\lambda}(x, t)\right|^{(\alpha-1) / n+1} \mathrm{~d} x \\
& \leqslant C R^{-1} t^{-1+1 / \alpha} .
\end{aligned}
$$

Moreover, a change of variables and properties of $\psi_{R}$ imply that 


$$
\left\|u_{0, \lambda} \psi_{R}\right\|_{1}=\int_{\mathbb{R}^{n}}\left|u_{0}(x)\right| \psi\left(\frac{x}{\lambda R}\right) \mathrm{d} x \leqslant \int_{|x| \geqslant \lambda R}\left|u_{0}(x)\right| \mathrm{d} x .
$$

Thus the integration of (3.31) over $[0, t]$, and the application of the above inequalities to the resulting equation, lead to the estimate

$$
\left|\int_{\mathbb{R}^{n}} u_{\lambda}(x, t) \psi_{R}(x) \mathrm{d} x\right| \leqslant C \int_{|x| \geqslant \lambda R}\left|u_{0}(x)\right| \mathrm{d} x+C\left(R^{-\alpha}+R^{-2}\right)+C R^{-1} t^{-1 / \alpha},
$$

for all $\lambda, t, R$, and a constant $C$. Since $u_{0} \in L^{1}\left(\mathbb{R}^{n}\right)$, the right hand side tends to 0 as $R \rightarrow \infty$, uniformly with respect to $\lambda \geqslant 1$. Hence, (3.30) holds true for $p=1$.

To prove the conclusion of Lemma 3.10 in the case $1<p<\infty$, it suffices to use the elementary interpolation inequality $\|v\|_{p} \leqslant\|v\|_{1}^{1 / p}\|v\|_{\infty}^{1-1 / p}$ with $v=u_{\lambda}(t) \psi_{R}$, and the bound $\left\|u_{\lambda}(t)\right\|_{\infty} \leqslant C t^{-n / \alpha}$ valid for all $t, \lambda$, and a constant $C$, already proved in (3.18).

\section{Self-similar source solutions - proof of Theorem 2.1}

The proof will follow the plan:

(i) To establish the existence of source solutions we apply an approximation argument based on solvability results proved in [6]. Namely, we approximate Eq. (2.1) by its parabolic regularization

$$
u_{t}+\mathcal{L} u+b \cdot \nabla\left(u|u|^{(\alpha-1) / n}\right)=0
$$

where $\mathcal{L}=-\varepsilon \Delta+(-\Delta)^{\alpha / 2}$ for small $\varepsilon>0$. At the same time the singular initial data (2.2) are also approximated by smooth positive functions $u_{0}^{\varepsilon}$ with compact supports shrinking to $\{0\}, u_{0}^{\varepsilon} \in L^{1}\left(\mathbb{R}^{n}\right) \cap L^{\infty}\left(\mathbb{R}^{n}\right)$, and $\int_{\mathbb{R}^{n}} u_{0}^{\varepsilon}(x) \varphi(x) \mathrm{d} x \rightarrow M \varphi(0)$ as $\varepsilon \rightarrow 0$.

(ii) For the proof of uniqueness of these solutions we adopt the ideas from [15, Section 3] and [10, proof of Theorem 1], where uniqueness of source solutions has been proved for an equation with $-\Delta$ as the diffusion operator and the convection term similar to that in (2.2). The first step is to show the result for nonnegative solutions.

(iii) Finally, the uniqueness result is extended to solutions $U$ of arbitrary sign.

(i) Proof of existence. We take as $u_{0}^{\varepsilon}$ the rescaled function $0 \leqslant \psi \in C_{c}^{\infty}\left(\mathbb{R}^{n}\right)$ with $\int_{\mathbb{R}^{n}} \psi(x) \mathrm{d} x=1$, that is

$$
u_{0}^{\varepsilon}(x)=\varepsilon^{-n} M \psi\left(\varepsilon^{-1} x\right) .
$$

In this setting, a unique, mild solution $u^{\varepsilon}$ has been constructed in [6, Section 3]. Now we prove the convergence of approximating solutions $u^{\varepsilon}$ to a self-similar source solution $U$.

First, for each $1 \leqslant p \leqslant \infty$ and $t>0$, the approximations $u^{\varepsilon}$ satisfy the estimates

$$
\left\|u^{\varepsilon}(t)\right\|_{p} \leqslant C t^{-n(1-1 / p) / \alpha}\left\|u_{0}^{\varepsilon}\right\|_{1},
$$

following from (3.18), see also [6, Corollary 3.2]. Note that the constant $C$ is independent of $\varepsilon$ here, since the cited result on the decay of solutions is obtained from 
the decay of the semigroup $\mathrm{e}^{-t(-\Delta)^{\alpha / 2}}$, and not that of $\mathrm{e}^{-t \mathcal{L}}$ (the latter is, in fact, better due to $\mathrm{e}^{-t \mathcal{L}}=\mathrm{e}^{\varepsilon t \Delta} \mathrm{e}^{-t(-\Delta)^{\alpha / 2}}$, cf. the proof of Lemma 3.4). Second, since $u_{0}^{\varepsilon}$ are uniformly bounded in $L^{1}\left(\mathbb{R}^{n}\right)$ as $\varepsilon \rightarrow 0$ the gradient estimate

$$
\left\|\nabla u^{\varepsilon}(t)\right\|_{p} \leqslant C t^{-n(1-1 / p) / \alpha-1 / \alpha}
$$

holds true also for each $1 \leqslant p \leqslant \infty$ and a constant $C$ independent of $\varepsilon$. This is a particular case of (3.20) in Lemma 3.6. Hence, we infer from the compactness Lemma 3.7 that there exists a limit function $U=\lim _{k \rightarrow \infty} u^{\varepsilon_{k}}$ such that

$$
U \in C\left(\left[t_{1}, t_{2}\right] ; W^{-2-\eta, 2}\left(\mathbb{R}^{n}\right)\right) \text { for all } 0<t_{1}<t_{2}<\infty \text { and each } \eta>0 .
$$

Then, by (4.2)-(4.3) and an evident extension of standard regularity theory for parabolic equations in [21, Chapter 3], we will see that $U \in C\left((0, \infty) ; L^{1}\left(\mathbb{R}^{n}\right)\right)$ $\cap L_{\text {loc }}^{\infty}\left((0, \infty) ; L^{\infty}\left(\mathbb{R}^{n}\right)\right)$ and further $U \in C\left((0, \infty) ; L^{p}\left(\mathbb{R}^{n}\right)\right) \cap L_{\text {loc }}^{\infty}\left((0, \infty) ; W^{2, p}\left(\mathbb{R}^{n}\right)\right)$.

In the last step of the existence proof we need to check that $U$ assumes the initial data (2.2) in the sense of measures; but this is a direct consequence of Lemmas 3.9 and 3.10.

(ii) Proof of uniqueness for positive initial data. Since the convection is unidirectional, the reasoning is, in principle, one-dimensional. Indeed, we may suppose, with no loss of generality, that $\mathbb{R}^{n} \ni b=(0, \ldots, 1)$. Denote $\mathbb{R}^{n} \ni x=\left(x_{1}, \ldots, x_{n-1}, x_{n}\right)$ by $\left(y, x_{n}\right)$, where $y=\left(x_{1}, \ldots, x_{n-1}\right)$ and $x_{n} \in \mathbb{R}$, so that (2.1) becomes

$$
u_{t}+(-\Delta)^{\alpha / 2} u+\frac{\partial}{\partial x_{n}}\left(u|u|^{(\alpha-1) / n}\right)=0 .
$$

We begin by proving that for two nonnegative solutions $U$ and $\bar{U}$ of the problem (4.4), (2.2), the equality

$$
\int_{-\infty}^{x_{n}} U(y, s, t) \mathrm{d} s=\int_{-\infty}^{x_{n}} \bar{U}(y, s, t) \mathrm{d} s
$$

holds for all $y \in \mathbb{R}^{n-1}$, and $x_{n} \in \mathbb{R}$; differentiation will imply that $U$ and $\bar{U}$ coincide. The key observation is that both functions

$$
\widetilde{U}(y, t)=\int_{-\infty}^{\infty} U(y, s, t) \mathrm{d} s \quad \text { and } \quad \widetilde{U}(y, t)=\int_{-\infty}^{\infty} U(y, s, t) \mathrm{d} s
$$

satisfy the linear equation

$$
v_{t}+(-\Delta)^{\alpha / 2} v=0
$$

in $\mathbb{R}^{n-1}$, with the initial data $\delta_{0}=\delta_{0}(y), y \in \mathbb{R}^{n-1}$. Indeed, the integration with respect to $x_{n}$ and the operator $(-\Delta)^{\alpha / 2}$ commute, which can be seen from the Fourier representation and the Lévy-Khintchine formula (1.3). Thus, $\widetilde{U}$ and $\widetilde{\bar{U}}$ are both equal to the multiple of $(n-1)$-dimensional fundamental solution $p_{\alpha}(y, t) \equiv \mathrm{e}^{-t(-\Delta)_{n-1}^{\alpha / 2}} \delta_{0}(y)$ 
of (4.6):

$$
\widetilde{U}(t)=\widetilde{\bar{U}}(t)=M p_{\alpha}(t) .
$$

Now consider an auxiliary function $w$ defined, for $x=\left(y, x_{n}\right), t$ and $r>0$, by

$$
w\left(y, x_{n}, t ; r\right) \equiv\left(U\left(y, x_{n}, t\right)-\frac{1}{2 r} \psi(y, t ; r)\right) \mathbb{1}_{[-r, r]}\left(x_{n}\right)
$$

where $\psi(y, t ; r) \equiv \int_{-r}^{r} U(y, s, t) \mathrm{d} s-p_{\alpha}(y, t)$. Observe that $w(y, \cdot, t ; r)$ is supported in the interval $[-r, r]$ for all $y \in \mathbb{R}^{n-1}$ and $t>0$, and $\int_{-\infty}^{\infty} w(y, s, t ; r) \mathrm{d} s=p_{\alpha}(y, t)$. Analogous properties are satisfied for the function $\bar{w}$ defined as in (4.8) with $U$ replaced by $\bar{U}$.

It is a consequence of Lemma 3.10 that

$$
\int_{\mathbb{R}^{n-1}} \int_{|s|>r}|U(y, s, t)| \mathrm{d} y \mathrm{~d} s \rightarrow 0 \quad \text { as } t \rightarrow 0 .
$$

Thus $\psi(y, t ; r)$ tends to 0 in $L^{1}\left(\mathbb{R}_{y}^{n-1}\right)$, as $t \rightarrow 0$, because

$$
\|\psi(\cdot, t ; r)\|_{1}=\int_{\mathbb{R}^{n-1}}\left|\int_{|s|>r} u(y, s, t) \mathrm{d} s\right| \mathrm{d} y .
$$

We consider now solutions $w^{k}$ and $\bar{w}^{k}, k=1,2,3, \ldots$, of the fractal Burgers equation (4.4) with the initial data $w^{k}\left(y, x_{n}, 1 / k ; r\right)$ and $\bar{w}^{k}\left(y, x_{n}, 1 / k ; r\right)$, respectively. These functions satisfy (by (4.7))

$$
\int_{-\infty}^{\infty} w^{k}(y, s, t ; r) \mathrm{d} s=\int_{-\infty}^{\infty} w^{k}(y, s, 1 / k ; r) \mathrm{d} s=p_{\alpha}(y, 1 / k)=\int_{-\infty}^{\infty} \bar{w}^{k}(y, s, t ; r) \mathrm{d} s .
$$

Define the (one-dimensional) potentials of $w^{k}$ and $\bar{w}^{k}$ by

$$
\begin{aligned}
& z^{k}\left(y, x_{n}, t ; r\right)=\int_{-\infty}^{x_{n}} w^{k}(y, s, t ; r) \mathrm{d} s, \\
& \bar{z}^{k}\left(y, x_{n}, t ; r\right)=\int_{-\infty}^{x_{n}} \bar{w}^{k}(y, s, t ; r) \mathrm{d} s .
\end{aligned}
$$

The support properties of $w(y, \cdot, 1 / k ; r), \bar{w}(y, \cdot, 1 / k ; r)$ imply that, for each $\left(y, x_{n}\right) \in$ $\mathbb{R}^{n}$ and $r>0$,

$$
z^{k}\left(y, x_{n}-2 r, 0 ; r\right) \leqslant \bar{z}^{k}\left(y, x_{n}, 0 ; r\right) \leqslant z^{k}\left(y, x_{n}+2 r, 0 ; r\right) .
$$


The crucial observation is that $z^{k}$ and $\bar{z}^{k}$ are nondecreasing with respect to $x_{n}$ and solve the equation

$$
z_{t}+(-\Delta)^{\alpha / 2} z+\left(\frac{\partial}{\partial x_{n}} z\right)^{1+(\alpha-1) / n}=0 .
$$

From the comparison principle in Lemma 3.1, this time for the functions $w=\partial z / \partial x_{n}$, we obtain the inequalities

$$
z^{k}\left(y, x_{n}-2 r, t ; r\right) \leqslant \bar{z}^{k}\left(y, x_{n}, t ; r\right) \leqslant z^{k}\left(y, x_{n}+2 r, t ; r\right) .
$$

Passing to the limit $k \rightarrow \infty$ in the above inequalities we get

$$
\int_{-\infty}^{x_{n}-2 r} U(y, s, t) \mathrm{d} s \leqslant \int_{-\infty}^{x_{n}} \bar{U}(y, s, t) \mathrm{d} s \leqslant \int_{-\infty}^{x_{n}+2 r} U(y, s, t) \mathrm{d} s .
$$

The passage to the limit in (4.10) is justified by noting that it suffices to show that

$$
\left\|w^{k}(\cdot, \cdot, t ; r)-U(t+1 / k)\right\|_{1} \rightarrow 0 \quad \text { as } k \rightarrow \infty,
$$

and the corresponding property for $\bar{w}^{k}, \bar{U}$. By the $L^{1}$-contraction property of solutions $u, \bar{u}$ of $(1.1):\|u(t)-\bar{u}(t)\|_{1} \leqslant\|u(0)-\bar{u}(0)\|_{1}$, proved in [6, (3.6)], we need to check (4.11) for $t=0$ only. That means

$$
\left\|U\left(y, x_{n}, 1 / k\right)\left(1-\mathbb{1}_{[-r, r]}\left(x_{n}\right)\right)+\frac{1}{2 r} \psi(y, 1 / k) \mathbb{1}_{[-r, r]}\left(x_{n}\right)\right\|_{1} \rightarrow 0 \quad \text { as } k \rightarrow \infty .
$$

The norm of the first term is

$$
\int_{\mathbb{R}^{n-1}} \int_{|s|>r}|w(y, s, 1 / k ; r)| \mathrm{d} y \mathrm{~d} s,
$$

which tends to 0 as $k \rightarrow \infty$ by the definition of the source solution. The norm of the second term is bounded from above by $\|\psi(\cdot, 1 / k ; r)\|_{1}$ (this term is in $L^{1}\left(\mathbb{R}_{y}^{n-1}\right)$ ) which also tends to 0 as $k \rightarrow \infty$, cf. (4.9). Since (4.10) holds for every $r>0$, we pass to the limit $r \rightarrow 0$ and arrive at (4.5). This concludes the proof of uniqueness of nonnegative source solutions of (2.1)-(2.2).

(iii) Proof of uniqueness for initial data of arbitrary sign. Since $U=U_{+}-U_{-}$and $\{U(t)\}_{t>0}$ is compact in the sense of narrow convergence of measures, both families $\left\{U_{+}(t)\right\}_{t>0}$ and $\left\{U_{-}(t)\right\}_{t>0}$ also are. Thus, for each sequence of $\tau>0$ tending to 0 , there exists a subsequence $\tau_{j} \rightarrow 0$ and two finite nonnegative measures $v_{+}, \nu_{-}$such that $M \delta_{0}=v_{+}-v_{-}$and $U_{+}\left(\tau_{j}\right) \rightarrow v_{+}, U_{-}\left(\tau_{j}\right) \rightarrow v_{-}$in the sense of measures as $j \rightarrow \infty$. By Lemma 3.10, the measures $v_{+}, v_{-}$are supported on $\{0\}$. Consequently, they are multiples of the Dirac measure $\delta_{0}$.

Consider a sequence $m_{j}$ of nonnegative functions in $C_{c}^{\infty}\left(\mathbb{R}^{n}\right)$ converging to $M \delta_{0}$, for instance defined as in (4.1) with $\varepsilon=1 / j, j=1,2,3, \ldots$. Let $g_{j}$ and $h_{j}$ be the solutions 
of (4.4) with the initial data at $t=0$ equal to $U_{+}\left(\tau_{j}\right)$ and $U_{-}\left(\tau_{j}\right)+m_{j}$, respectively. It follows from the comparison principle in Lemma 3.1, oddness of $f(s)=s|s|^{(\alpha-1) / n}$ and uniqueness of solutions (3.4), that

$$
0 \leqslant U_{+}\left(t+\tau_{j}\right) \leqslant g_{j}(t), \quad 0 \leqslant U_{-}\left(t+\tau_{j}\right) \leqslant h_{j}(t) .
$$

The functions $g_{j}$ and $h_{j}$ solve the problem (4.4), (2.2) with the initial data in $L^{1}\left(\mathbb{R}^{n}\right) \cap$ $L^{\infty}\left(\mathbb{R}^{n}\right)$. The proof that $0 \leqslant g_{j}$ and $0 \leqslant h_{j}$ converge to some positive functions $g$ and $h$ which solve (2.1) in the sense of distributions, consists of the steps essentially described above for the approximations $u^{\varepsilon}$ of $U$. Since the necessary modifications are evident, we skip the details.

The limit functions $g$ and $h$ take on the initial data $M \delta_{0}$ in the sense of measures which is again based on the Lemma 3.10. Therefore, $g$ and $h$ are positive solutions of the problem (4.4), (2.2), so the first part of the proof applies, and $g \equiv h$. Passing to the limit $j \rightarrow \infty$ in (4.12) we have

$$
U_{+}(t)+U_{-}(t) \leqslant g(t)=h(t),
$$

and letting $t$ tend to 0

$$
M \delta_{0}+v_{-} \leqslant M \delta_{0},
$$

that is, $v_{-}=0$. To conclude, observe that $U_{-}$(as well as $U_{+}$) is a nonnegative subsolution of (4.4) with 0 as the initial value

$$
\frac{\partial}{\partial t} U_{-}+(-\Delta)^{\alpha / 2} U_{-}+\frac{\partial}{\partial x_{n}}\left(U_{-}\left|U_{-}\right|^{(\alpha-1) / n}\right) \leqslant 0 .
$$

This can be seen from the Kato-Beurling-Deny inequalities, cf. [11, (1.3.1) and (1.3.2)]. Indeed, we have

$$
\mathcal{L}(|v|) \leqslant \frac{v}{|v|} \mathcal{L}|v|
$$

in the sense of distributions (the precise meaning of this inequality is: $\left\langle\mathcal{L}^{1 / 2} \varphi, \mathcal{L}^{1 / 2}|v|\right\rangle \leqslant$ $\left\langle\varphi, v|v|^{-1} \mathcal{L} v\right\rangle$ for each $0 \leqslant \varphi$ and $v$ in the domain of the quadratic form on $L^{2}\left(\mathbb{R}^{n}\right)$ associated with $\mathcal{L}$ ). Consequently, we get

$$
\mathcal{L}\left(U_{-}\right)=\frac{1}{2}(\mathcal{L}(|U|)-\mathcal{L} U) \leqslant \frac{1}{2}\left(\frac{U}{|U|} \mathcal{L}(U)-\mathcal{L}(U)\right)=\left(\operatorname{sgn} U_{-}\right) \mathcal{L} U,
$$

and, similarly, $\mathcal{L}\left(U_{+}\right) \leqslant\left(\operatorname{sgn} U_{+}\right) \mathcal{L} U$. Then, taking into account the oddness of the nonlinear term, it follows that

$$
\frac{\partial}{\partial t}\left(U_{-}\right)+\mathcal{L} U_{-}+\frac{\partial}{\partial x_{n}}\left(U_{-}\left|U_{-}\right|^{(\alpha-1) / n}\right) \leqslant 0
$$

in the sense of distributions on $\mathbb{R}^{n} \times(0, \infty)$.

Then $0 \leqslant v(y)=\int_{-\infty}^{\infty} U_{-}\left(y, x_{n}\right) \mathrm{d} x_{n}$ is a subsolution of the linear equation (4.6) in $\mathbb{R}^{n-1}$ with 0 as the initial value, so $U_{-} \equiv 0$. Therefore, $U=U_{+}$, and, by the first part of the proof, $U=U_{M}$. 
Remark 4.1. - The self-similar solution $U$ decays algebraically in the space variable $x$, so that $|U(x, 1)| \leqslant C(1+|x|)^{-(n+\alpha)}$, cf. [20] for related estimates of $p_{\alpha}(t)$ and generalizations for $\mathrm{e}^{-t \mathcal{L}}$. This is, of course, in contrast with the Gaussian decay of $U$ in $x$ for (1.4).

\section{Self-similar asymptotics - proof of Theorem 2.2}

The crucial observation in the proof of Theorem 2.2 is that the investigation of the asymptotic behavior of a solution $u$ can be reduced to studying the convergence of the family $\left\{u_{\lambda}\right\}_{\lambda>0}$ as $\lambda \rightarrow \infty$. Indeed, if we note that

$$
u_{\lambda}(x, 1)-U(x, 1)=\lambda^{n}\left(u\left(\lambda x, \lambda^{\alpha}\right)-U\left(\lambda x, \lambda^{\alpha}\right)\right),
$$

then choosing $\lambda^{\alpha}=t$ we have

$$
\left\|u_{\lambda}(1)-U(1)\right\|_{p}=\lambda^{n(1-1 / p)}\left\|u\left(\lambda^{\alpha}\right)-U\left(\lambda^{\alpha}\right)\right\|_{p}=t^{n(1-1 / p) / \alpha}\|u(t)-U(t)\|_{p} .
$$

Thus, the convergence in the $L^{p}$-norm of $u_{\lambda}(x, 1)$ to $U(x, 1)$ as $\lambda \rightarrow \infty$ is equivalent to (2.10). Of course, the same is true, if we replace $t=1$ by any fixed $t_{0}>0$.

From this point on, the proof is based on lemmas proved in Section 3.

(i) First, using estimates of the family $\left\{u_{\lambda}\right\}_{\lambda \geqslant 1}$ uniform with respect to $\lambda$ from Lemma 3.5 and 3.6 and compactness arguments from Lemma 3.7, we find $\bar{u}$ and a sequence $\lambda_{k} \rightarrow \infty$ such that (3.22)-(3.25) hold true. By Lemma 3.8, the function $\bar{u}$ satisfies the Eq. (2.1) in the sense of distributions.

(ii) Next, combining Lemmatas 3.9 and 3.10, we arrive at $\bar{u}(x, 0)=M \delta_{0}$. This implies, by the uniqueness of $U=U_{M}$ proved in Theorem 2.1, that $\bar{u}(x, t)=U(x, t)$ and $u_{\lambda} \rightarrow U$ as $\lambda \rightarrow \infty$.

(iii) Finally, Lemma 3.10 and (3.27) imply that the convergence of $u_{\lambda}(t)$ toward $U(t)$ takes place not only in the local or weak sense (cf. Lemma 3.7), but actually in $L^{p}\left(\mathbb{R}^{n}\right)$ for each $1 \leqslant p<\infty$.

The proof of (2.10) for $p=\infty$ requires another argument involving the integral equation (2.3). Recall that $\int_{\mathbb{R}^{n}} u(x, t) \mathrm{d} x=M$ and $U=U_{M}$ is the corresponding source solution of (2.2). A calculation involving (2.3) yields the following identity

$$
\begin{aligned}
& u(t+1)-U(t+1)=\mathrm{e}^{-t \mathcal{L}}(u(1)-U(1)) \\
& -\int_{0}^{t} b \cdot \nabla \mathrm{e}^{-(t-\tau) \mathcal{L}}\left(u|u|^{(\alpha-1) / n}-U|U|^{(\alpha-1) / n}\right)(\tau+1) \mathrm{d} \tau .
\end{aligned}
$$

Since $\int_{\mathbb{R}^{n}}(u(x, 1)-U(x, 1)) \mathrm{d} x=0$, it follows from Corollary 3.1 that

$$
t^{n / \alpha}\left\|\mathrm{e}^{-t \mathcal{L}}(u(1)-U(1))\right\|_{\infty} \rightarrow 0 \quad \text { as } t \rightarrow \infty .
$$

We split the integration range with respect to $\tau$ in the second term on the right hand side of (5.2) into $[0, t / 2]$ and $[t / 2, t]$. For $\tau \in[0, t / 2]$ the $L^{\infty}$-norm of the integrand is bounded by 


$$
\begin{aligned}
\| b \cdot \nabla & \left(\mathrm{e}^{-(t-\tau) \mathcal{L}}\right)\left\|_{\infty}\right\| u(\tau+1)-U(\tau+1) \|_{1} \\
\quad & \times\left(\|u(\tau+1)\|_{\infty}^{(\alpha-1) / n}+\|U(\tau+1)\|_{\infty}^{(\alpha-1) / n}\right) \\
\leqslant & C(t-\tau)^{-n / \alpha-1 / \alpha} \tau^{-1+1 / \alpha}\|u(\tau+1)-U(\tau+1)\|_{1} .
\end{aligned}
$$

Hence, by the Lebesgue Dominated Convergence Theorem and (2.10) with $p=1$, we have

$$
\begin{aligned}
& t^{\alpha / n}\left\|\int_{0}^{t / 2} b \cdot \nabla \mathrm{e}^{-(t-\tau) \mathcal{L}}\left(u|u|^{(\alpha-1) / n}-U|U|^{(\alpha-1) / n}\right)(\tau+1) \mathrm{d} \tau\right\|_{\infty} \\
& \quad \leqslant C t^{n / \alpha} \int_{0}^{t / 2}(t-\tau)^{-n / \alpha-1 / \alpha} \tau^{-1+1 / \alpha}\|u(\tau+1)-U(\tau+1)\|_{1} \mathrm{~d} \tau \\
& \quad \leqslant C \int_{0}^{1 / 2}(1-s)^{-n / \alpha-1 / \alpha} s^{-1+1 / \alpha}\|u(t s+1)-U(t s+1)\|_{1} \mathrm{~d} s \rightarrow 0
\end{aligned}
$$

as $t \rightarrow \infty$.

We proceed analogously with the integral on the right hand side of (5.2) for $\tau \in[t / 2, t]$. However, in this case, it is necessary to modify the inequality (5.3) in the following way. By the Hölder inequality, for every $p, q \in[1, \infty]$ satisfying $1 / p+1 / q=1$, we have the $L^{\infty}$-norm of the integrand in (5.2) bounded from above by

$$
\begin{aligned}
\| b \cdot \nabla & \left(\mathrm{e}^{-(t-\tau) \mathcal{L}}\right)\left\|_{q}\right\| u(\tau+1)-U(\tau+1) \|_{p} \\
& \times\left(\|u(\tau+1)\|_{\infty}^{(\alpha-1) / n}+\|U(\tau+1)\|_{\infty}^{(\alpha-1) / n}\right) \\
\leqslant & C(t-\tau)^{-n /(p \alpha)-1 / \alpha}(\tau+1)^{-1+1 / \alpha-n(1-1 / p) / \alpha} \\
& \times\left((\tau+1)^{n(1-1 / p) / \alpha}\|u(\tau+1)-U(\tau+1)\|_{p}\right) .
\end{aligned}
$$

From now on, we repeat the reasoning in the case $\tau \in[0, t / 2]$ provided $q \in(1, \infty)$ is chosen so that $-n /(p \alpha)-1 / \alpha>-1$, i.e., $p>n /(\alpha-1)$. This concludes the proof of Theorem 2.2.

Remark 5.1. - Assumption (2.6) on the operator $\mathcal{L}$ can be replaced by weaker assumptions

$$
\lim _{\xi \rightarrow 0} \frac{a(\xi)}{|\xi|^{\alpha}} \in(0, \infty) \quad \text { and } \quad \inf _{\xi \in \mathbb{R}^{n}} \frac{a(\xi)}{|\xi|^{\alpha}}>0 .
$$

In this case the proof of the crucial decay estimates (3.2) and (3.15) in Lemma 3.4 necessitates a supplementary smoothness assumption on $a$ for $\xi \neq 0$.

Remark 5.2. - The result in Theorem 2.2 remains true if we the nonlinear term $f(s)$ in Eq. (1.1) just enjoys the correct critical asymptotics at 0 , that is, if it satisfies the condition $\lim _{s \rightarrow 0} f(s) /\left(s|s|^{(\alpha-1) / n}\right) \in(0, \infty)$. 


\section{Acknowledgements}

This research has been begun while the first and the second named authors were research scholars at the CWRU Center for Stochastic and Chaotic Processes in Science and Technology, Cleveland (Summer 1999). Grant support from NSF 97-04231, KBN 324/P03/97/12, 50/P03/2000/18 and POLONIUM 98111 is gratefully acknowledged. We thank Ana Carpio for sending us her paper, and Thierry Coulhon, Jacek Dziubański and Jacek Zienkiewicz for interesting comments.

\section{REFERENCES}

[1] Bardos C., Penel P., Frisch U., Sulem P.L., Modified dissipativity for a nonlinear evolution equation arising in turbulence, Arch. Rat. Mech. Anal. 71 (1979) 237-256.

[2] Bertoin J., Lévy Processes, Cambridge University Press, 1996.

[3] Biler P., Funaki T., Woyczynski W.A., Fractal Burgers equations, J. Differential Equations 148 (1998) 9-46.

[4] Biler P., Funaki T., Woyczyński W.A., Interacting particle approximation for nonlocal quadratic evolution problems, Prob. Math. Stat. 19 (1999) 267-286.

[5] Biler P., Karch G., Woyczynski W.A., Asymptotics for multifractal conservation laws, Studia Math. 135 (1999) 231-252.

[6] Biler P., Karch G., Woyczyński W.A., Asymptotics for conservation laws involving Lévy diffusion generators, preprint.

[7] Biler P., Karch G., Woyczyński W.A., Multifractal and Lévy conservation laws, C. R. Acad. Sci. Paris, Sér. I Math. 330 (2000) 343-348.

[8] Biler P., Woyczyński W.A., Global and exploding solutions for nonlocal quadratic evolution problems, SIAM J. Appl. Math. 59 (1999) 845-869.

[9] Carpio A., Asymptotic behavior for the vorticity equations in dimensions two and three, Comm. PDE 19 (1994) 827-872.

[10] Carpio A., Large time behaviour in some convection-diffusion equations, Ann. Sc. Norm. Sup. Pisa, ser. IV, 23 (1996) 551-574.

[11] Davies E.B., Heat Kernels and Spectral Theory, Cambridge University Press, 1989.

[12] Duoandikoetxea J., Zuazua E., Moments, masses de Dirac et décomposition de fonctions, C. R. Acad. Sci. Paris, Sér. I Math. 315 (1992) 693-698.

[13] Duro G., Zuazua E., Large time behavior for convection-diffusion equations in $\mathbb{R}^{N}$ with asymptotically constant diffusion, Comm. PDE 24 (1999) 1283-1340.

[14] Escobedo M., Vázquez J.L., Zuazua E., Asymptotic behaviour and source-type solutions for a diffusion-convection equation, Arch. Rat. Mech. Anal. 124 (1993) 43-65.

[15] Escobedo M., Vázquez J.L., Zuazua E., A diffusion-convection equation in several space dimensions, Indiana Univ. Math. J. 42 (1993) 1413-1440.

[16] Escobedo M., Zuazua E., Large time behavior for convection-diffusion equations in $\mathbb{R}^{N}$, J. Funct. Anal. 100 (1991) 119-161.

[17] Escobedo M., Zuazua E., Long-time behaviour of diffusion waves for a viscous system of conservation laws in $\mathbb{R}^{N}$, Asymptotic Analysis 20 (1999) 133-173.

[18] Jacob N., Pseudo-Differential Operators and Markov Processes, Akademie Verlag, Berlin, 1996.

[19] Karch G., Self-similar large time behavior of solutions to Korteweg-de Vries-Burgers equation, Nonlinear Analysis 35 (1999) 199-219. 
[20] Komatsu T., Uniform estimates for fundamental solutions associated with non-local Dirichlet forms, Osaka J. Math. 32 (1995) 833-860.

[21] Ladyženskaja O.A., Solonnikov V.A., Ural'ceva N.N., Linear and Quasilinear Equations of Parabolic Type, Amer. Math. Soc., Providence, RI, 1988.

[22] Mann J.A., Jr., Woyczynski W.A., Growing fractal interfaces in the presence of self-similar hopping surface diffusion, Physica A 291 (2001) 159-183.

[23] Pȩkalski A., Sznajd-Weron K. (Eds.), Anomalous Diffusion. From Basics to Applications, Lecture Notes in Physics, Vol. 519, Springer-Verlag, Berlin, 1999.

[24] Shlesinger M.F., Zaslavsky G.M., Frisch U. (Eds.), Lévy Flights and Related Topics in Physics, Lecture Notes in Physics, Vol. 450, Springer-Verlag, Berlin, 1995.

[25] Simon J., Compact sets in the space $L^{p}(0, T ; B)$, Annali Mat. Pura Appl. 156 (1987) 65-96.

[26] Stroock D.W., Diffusion processes associated with Lévy generators, Z. Wahr. verw. Geb. 32 (1975) 209-244.

[27] Woyczynski W.A., Burgers-KPZ Turbulence - Göttingen Lectures, Lecture Notes in Math., Vol. 1700, Springer-Verlag, Berlin, 1998.

[28] Zuazua E., Weakly nonlinear large time behavior in scalar convection-diffusion equations, Differential Integral Equations 6 (1993) 1481-1491. 